\title{
Cambio climático. Perspectivas futuras
}

\author{
David GalÁN MADRUGA ${ }^{1,2}$ \\ José Luis GARrido Morales ${ }^{1}$ \\ david.galan@isciii.es
}

Recibido: 18 de mayo del 2012

Enviado a evaluar: 24 de mayo del 2012

Aceptado: 27 de julio del 2012

\section{RESUMEN}

El presente artículo pretende ofrecer una visión global referente al cambio climático en relación a aspectos tales como su relación con los gases de efecto invernadero, efectos sobre el medioambiente, ser humano, economía, así como las posibles consecuencias futuras y la importancia en la toma de decisiones y políticas encaminadas a mitigar el calentamiento a nivel mundial.

Palabras clave: Cambio climático, gases de efecto invernadero y calentamiento global.

\section{Climatic change. Future perspectives}

\begin{abstract}
The present article aims to offer an overview of climate change in relation to aspects such as their relationship to greenhouse gases (GHG), effects on the environment, human being, economy, possible consequences in a future and the importance in the policies and decision taken and to mitigate global warming.
\end{abstract}

Keywords: Climatic change, greenhouse gases and global warming.

1 Área de Contaminación Atmosférica. Centro Nacional de Sanidad Ambiental. Instituto de Salud Carlos III Ctra. de Majadahonda a Pozuelo, km2,2. 28220 Majadahonda (Madrid)

2 Escala de Científicos Titulares de OPIs. Responsable de la Unidad de Laboratorio de Cromatografía de Gases/Espectrometría de Masas 


\section{INTRODUCCIÓN}

\subsection{DEFINICIÓN}

Según la National Oceanic and Atmospheric Administration (NOAA) el cambio climático responde a fluctuaciones a largo plazo de la temperatura, las precipitaciones, los vientos y todas los demás componentes del clima en la Tierra.

Naciones Unidas define el Cambio Climático como un cambio en el clima, atribuible directa o indirectamente a la actividad humana, que altera la composición de la atmósfera mundial y que se suma a la variabilidad climática natural observada durante períodos de tiempo comparables.

\subsection{ANTECEDENTES}

La primera Conferencia Mundial sobre el Clima en el mundo tuvo lugar en Génova en 1979, donde por primera vez se debatió el problema del cambio climático en la comunidad científica (WMO, 1979). Una de las consecuencias de dicha declaración fue la posterior aparición de una serie de conferencias científicas y políticas: Villach, Toronto, (Conference Statement, 1988), Ottawa, Tata, La Haya, Noordwijk, El Cairo y Bergen (1989) y la Segunda Conferencia Mundial sobre el Clima (1990). En este año se creo el IPCC, Grupo Intergubernamental de Expertos sobre el Cambio Climático, y tuvo lugar la primera Asamblea General de la ONU sobre cambio climático. El IPCCC fue creado por el PNUMA (Programa de las Naciones Unidas para el Medio Ambiente) y la OMM (Organización Meteorológica Mundial). En su primer informe, el IPCC identificó como gases de efecto invernadero (GEI) el dióxido de carbono $\left(\mathrm{CO}_{2}\right)$, metano $\left(\mathrm{CH}_{4}\right)$, óxido nitroso $\left(\mathrm{N}_{2} \mathrm{O}\right)$ y los compuestos clorofluorocarbonos (CFC) (IPCC, 1990). En 1990 tuvo lugar la segunda Conferencia Mundial sobre el Clima.

La Convención del cambio climático de 1992 tenía por objetivo principal, por un lado, prevenir la interferencia antropogénica en el sistema climático y, por otro lado, permitir el desarrollo sostenible (Convention on climate change, 1992).

En 1996, se publicó el segundo informe de IPCC, donde las evidencias del estudio ponían de manifiesto una relación directa entre la influencia humana y el cambio climático (IPCC, 1995). En dicho informe apareció por primera vez el debate coste social - cambio climático (IPCC, 1995b).

En 1997, se desarrolló el Protocolo de Kyoto. Entre otros aspectos, dicho protocolo incluye, por un lado, políticas de eficiencia energética, forestación y reforestación, agricultura sostenible, la investigación y el uso de energías renovables, así como fomentar reformas en el sector transporte y control de las emisiones de metano (Gupta, 2010). Por otro lado, incluyen objetivos obligatorios relativos a las emisiones de GEI para las principales economías mundiales que lo hayan aceptado. Estos objetivos van desde $-8 \%$ hasta $+10 \%$ del nivel de emisión de los diferentes países en 1999 "con miras a reducir el total de sus emisiones, para esos gases, a un nivel 
inferior al 5\% con respecto al de 1990, y para un período de compromiso comprendido entre el año 2008 y el 2012.

El Tercer Informe de Evaluación de IPCC confirmó los resultados científicos de los informes anteriores, indicando que la mayor parte del calentamiento global observado durante los últimos 50 años era probable se debiera al aumento de las concentraciones de GEI (IPCC, 2001).

En 2007, en Bali, se adopta una decisión sobre los recursos necesarios para mantener el cambio climático dentro de los límites de seguridad.

El Cuarto Informe de Evaluación del IPCC afirma que las concentraciones globales de GEI han aumentado enormemente. El IPCC recomienda la energía nuclear como una de las tecnologías clave para la mitigación del calentamiento global.

\subsection{ESTADO ACTUAL}

Actualmente, existentes suficientes evidencias científicas que sitúan al cambio climático como el principal problema medioambiental del siglo XXI (WHO, 2008). Existe una asociación entre el cambio climático y la actividad antropogénica (McMichael, Friel, Nyong y Corvalán, 2008).

La relación entre cambio climático y actividad antropogénica es directa y convincente. La superficie de la Tierra se ha calentado más de $0,8^{\circ} \mathrm{C}$ durante el siglo pasado, y en aproximadamente $0,6{ }^{\circ} \mathrm{C}$ en las últimas tres décadas (CampbellLendrum, Corvalán y Neira, 2007).

En España, la Estrategia Española de Cambio Climático y Energía Limpia en el horizonte 2007 - 2012 - 2020, aprobada en octubre de 2007 por el Consejo Nacional del Clima y en noviembre en el Consejo de Ministros, pone de manifiesto, entre otros aspectos, respectar el compromiso adquirido por España con la ratificación del Protocolo de Kyoto y la adaptación al cambio climático como máximas prioridades.

Existe diferente legislación enfocada a las emisiones de GEI.

El Real Decreto 837/2002 regula la información relativa al consumo de combustible y a las emisiones de $\mathrm{CO}_{2}$ de los turismos nuevos que se pongan a la venta o se ofrezcan en arrendamiento financiero en territorio español.

El Real Decreto 1866/2004 aprueba el Plan Nacional de Asignación de derechos de emisión de gases de efecto invernadero, 2005-2007.

El Real Decreto 1264/2005, de 21 de octubre, por el que se regula el registro nacional de derechos de emisión de gases de efecto invernadero.

El Real Decreto 1370/2006 aprueba el Plan Nacional de Asignación de derechos de emisión de gases de efecto invernadero, 2008-2012.

\section{GASES DE EFECTO INVERNADERO}

En condiciones normales, la radiación solar calienta la superficie de la Tierra y parte de esa energía es emitida desde la Tierra hacia la atmósfera en forma de radiación infrarroja. Este equilibrio se basa en el balance energético de la Tierra, permitiendo mantener la temperatura terrestre en un margen de valores aptos para la vida 
(Erickson, 1992). Por tanto, toda variación de dicho equilibrio, ya sea de origen natural o antropogénico, supone un cambio en el clima.

Los denominados GEI retienen parte de la radiación infrarroja emitida desde la Tierra a la atmósfera, produciendo un efecto invernadero. Como consecuencia se produce un aumento de la temperatura en la superficie de la Tierra, produciendo el denominado calentamiento global. Por tanto, a mayor cantidad de GEI mayor será el aumento de temperatura superficial en la Tierra. Así, cuanto más rápido cambie el clima, mayor será el riesgo de daños. El sistema climático debe ajustarse al aumento de los GEI para así mantener el equilibrio de energía.

Como GEI, normalmente se engloban el dióxido de carbono $\left(\mathrm{CO}_{2}\right)$, metano $\left(\mathrm{CH}_{4}\right)$, óxido nitroso $\left(\mathrm{N}_{2} \mathrm{O}\right)$ y los compuestos clorofluorocarbonos (CFC).

A excepción de los compuestos CFC, el resto de compuestos son naturales, es decir, existen en la atmósfera desde antes de la aparición de los seres humanos. Sin embargo, a partir de mediados del siglo XIX, coincidiendo con la revolución industrial y con el aumento del uso de combustibles fósiles derivado de las actividades industriales y de transporte, se han producido notables incrementos en las emisiones de óxidos de nitrógeno y dióxido de carbono a la atmósfera. En igual medida, los niveles de metano están aumentado a nivel atmosférico debido a razones antropogénicas.

Además, a este incremento de emisiones, existen otros aspectos como la deforestación, que han reducido la cantidad de dióxido de carbono retenida en materia orgánica, contribuyendo así indirectamente al aumento antropogénico del efecto invernadero.

Los GEI presentan una larga permanencia en la atmósfera, como consecuencia de ello los efectos nocivos que su presencia en la atmósfera pueden provocar no son de efecto inmediato (Clima, 2001).

Como consecuencia, una reducción inmediata y drástica de las emisiones de GEI no impediría los efectos del cambio climático.

\section{CONSECUENCIAS FUTURAS}

\subsection{POSIBLES EFECTOS DERIVADOS DEL CAMBIO CLIMÁTICO: MEDIOAMBIENTE}

Las consecuencias derivadas del cambio climático se producen a nivel transfronterizo. Entre los diferentes efectos que pueden aparecer como consecuencia del cambio climático, en condiciones extremas, destaca un mayor riesgo de fenómenos meteorológicos extremos, tales como inundaciones, tormentas torrenciales de gran intensidad y con mayor frecuencia, cambios en las condiciones de sequía a largo plazo en muchas regiones, olas de calor mortales, etc.

El derretimiento de los glaciares, que abastecen de agua dulce a grandes centros de población, provoca un aumento del nivel del mar que conduce a la salinización de las fuentes de la agricultura y el agua potable. En igual medida, el nivel del mar ha aumentado 1,8 mm por año desde 1961, aunque en los últimos años la tasa de aumento es mucho mayor $(3,1 \mathrm{~mm})$ (IPCC, 2007). 
Dado que las pautas climáticas podrían variar como consecuencia del cambio climático, la agricultura mundial se vería afectada enormemente por los cambios de dichas pautas. Un aumento de la temperatura mundial inferior a $2,5{ }^{\circ} \mathrm{C}$ podría no tener efectos notables sobre la producción de alimentos a nivel mundial, un aumento superior a $2,5^{\circ} \mathrm{C}$ podría disminuir directamente la producción alimentaria, produciendo un aumento de hambruna. Por ello, cambiarían las pautas de producción (Cambio climático, 2004).

Actualmente, las emisiones de $\mathrm{CO}_{2}$ siguen aumentando, y los modelos climáticos proyectan, pasado el siglo XXI (Climate change, 2007), que la temperatura media de la superficie se incremente en un intervalo que abarca desde $1,1{ }^{\circ} \mathrm{C}$ a 6,4 ${ }^{\circ} \mathrm{C}$. Un estudio reciente del IPCC estima que para el año 2100 la temperatura superficial aumentará en un valor promedio de entre $1{ }^{\circ} \mathrm{C}$ y $3,5^{\circ} \mathrm{C}$, con una elevación asociada del nivel del mar de 15 a $95 \mathrm{~cm}$.

\subsection{POSIBLES EFECTOS DERIVADOS DEL CAMBIO CLIMÁTICO: SER HUMANO}

La salud humana se verá afectada a largo plazo por los efectos del cambio climático. El ser humano necesita de una serie de factores, tales como suficientes alimentos, agua potable, unas correctas condiciones sociales y ambientales para el control de enfermedades infecciosas, para disponer de una correcta salud. Los cambios en el clima afectarán a los factores de salud pública.

Unas de las consecuencias más importantes del cambio climático a largo plazo es el aumento en la frecuencia o intensidad de episodios meteorológicos extremos. Episodios de calor extremo provocarán enfermedades cardiovasculares y respiratorias.

Existen otros efectos potencialmente más graves que pueden afectar en la dinámica de enfermedades infecciosas. Desde el punto de vista sanitario, las repercusiones del cambio climático son potencialmente enormes. Muchas de las causas de muerte más importantes en el hombre son muy sensibles a las condiciones climáticas, tales como la malaria y la diarrea (WHO, 2004). El cambio climático afectaría a la distribución de las enfermedades a nivel mundial, afectando por ejemplo a los vectores de transmisión de dichas enfermedades.

Estas consecuencias futuras son consecuencia de la actividad humana, principalmente de actividades relacionadas con la industria y el transporte. Un aspecto importante para la mitigación de estas consecuencias sería la implicación de la sociedad para la mejora de esta situación (Galán Madruga, 2009). El papel del ciudadano resulta básico y esencial en la construcción del capital social que desemboque en políticas globales enfocadas a un cambio cultural y productivo respecto a los futuros efectos del cambio climático (Sunyer, 2012).

\subsection{POSIBLES EFECTOS DERIVADOS DEL CAMBIO CLIMÁTICO: ACTIVIDAD ECONÓMICA}

Como consecuencia de la aparición de periodos de precipitación y sequía extremos, la producción agrícola de alimentos a nivel mundial se verá afectada, cambian- 
do tanto el volumen de producción como la pauta de producción. El precio de los alimentos podría sufrir un aumento de valor dada el efecto adverso del calentamiento global sobre los cultivos a nivel mundial (Costello, Abbas, Allen, Ball, Bell, Bellamy, Friel, Groce, Johnson, Kett, Lee, Levy, Maslin, McCoy, McGuire, Montgomery, Napier, Pagel, Patel, Puppim de Oliveira, Redclift, Rees, Rogger, Scott, Stephenson, Twigg, Wolff y Patterson, 2009).

Las infraestructuras existentes dentro de los asentamientos humanos se verán afectados por episodios de precipitaciones extremas, dando lugar a inundaciones urbanas, a terremotos, dando lugar a deslizamientos de terrenos, etc...

Las poblaciones costeras, puertos, industria, se verán afectados por el aumento del nivel del mar.

En igual medida, la demanda energética por parte de la población mundial se verá aumentada enormemente, como consecuencia, por ejemplo, de la aparición de olas de calor extremo.

Figura 1: Visión esquemática de la problemática del cambio climático

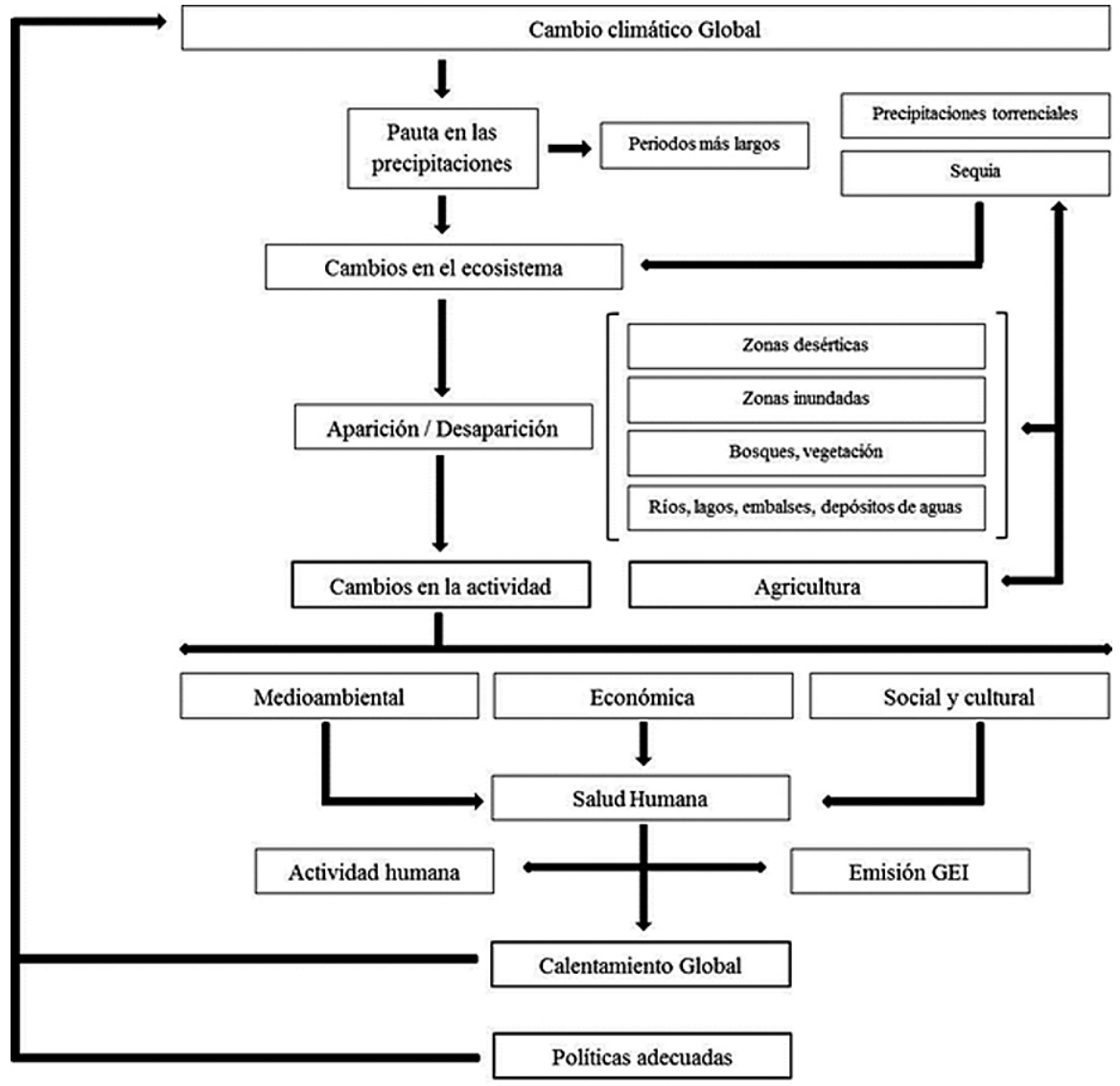


A continuación, en la Figura 1, se muestra una visión global del efecto del cambio climático.

\section{CONCLUSIONES}

El cambio climático se ha convertido en el gran problema ambiental del siglo XXI a nivel mundial, puesto que las consecuencias futuras, derivadas del calentamiento global, sobre el ser humano y el medioambiente no responden a criterios de fronteras de Estados, sino que se trata de un problema transfronterizo.

Para controlar o reducir en lo posible los efectos negativos derivados de dicho problema, es necesario llegar a un acuerdo, que no debe estar sujeto a la voluntad de los Países desarrollados, enfocado a aceptar medidas de largo alcance que permitan la reducción de emisiones de GEI que mitiguen el calentamiento global. La solución, a este problema Global, requiere una estrategia de dimensión internacional, a nivel estatal, regional y local y deben ser estrategias multidisciplinarias y multisectoriales.

El hombre y el medioambiente deberán adaptarse a las nuevas condiciones climáticas. La vulnerabilidad no sólo depende de la sensibilidad de la Tierra, sino también de su capacidad para adaptarse a nuevas condiciones climáticas.

Un aspecto básico, en la mitigación de la velocidad de calentamiento de la Tierra, es la elección de las energías a utilizar por parte del ser humano, así como la necesidad de proponer medidas que impliquen la reducción de la cantidad de energía consumida, traducidas en políticas energéticas, lo que proporcionará en una reducción de los denominados GEI y se evitará el progresivo calentamiento de la superficie de la Tierra.

De no mitigar las emisiones de GEI la ser humana tendrá que hacer frente a nuevos riesgos y presiones. Aspectos económicos, ambientales, sociales y culturales, así como la salud humana experimentarán efectos directos e indirectos de una enorme magnitud, lo cuál justifica la necesidad de estabilizar las concentraciones de GEI a nivel atmosférico.

\section{BIBLIOGRAFÍA}

Cambio climático (2004): Carpeta de información. Secretaría de la Convención sobre el Cambio Climático. Publicado por Naciones Unidas, PNUMA, OMM, OMS, UNITAR, UNFCCC.

CAMPBELL-LENDRUM, D., CORVALÁN, C. Y NEIRA, M. (2007): “Global climate change: implications for international public health policy". Bulletin of the World Health Organization. Vol 85 (3), 161 - 244.

Clima (2001): La base científica. Resumen técnico del Informe del Grupo de Trabajo I., p.38.

Climate Change (2007): The physical science basis: summary for policymakers. Geneva: Intergovernmental Panel on Climate Change secretariat.

Conference Statement (1998): Conference on The Changing Atmosphere: Implications for Global Security, organized by the Government of Canada, Toronto, June 27-30. 
Convention on Climate Change (1992). United Nations 31 ILM 849.

COSTELLO, A.; ABBAS, M.; ALLEN, A.; BALL, S.; BELL, S.; BELLAMY, R.; FRIEL, S.; GROCE, N.; JOHMSON, A.; KETT, M.; LEE, M.; LEVY, C.; MASLIN, M., MCCOY, D.; MCGUIRE, B.; MONTGOMERY, H.; NAPIER, D.; PAGEL, C.; PATEL, J.; PUPPIM DE OLIVEIRA, J.A.; REDCLIFT, N.; REES, H.; ROGGER, D.; SCOTT, J.; STEPHENSON, J.; TWIGG, J.; WOLFF , J. Y PATTERSON, C. (2009): "Managing the health effects of climate change". Lancet. Vol. 373, 1693 - 1733.

ERICKSON, J. (1992), El efecto invernadero. Ed. McGraw-Hill. 1992. 217 páginas GALAN MADRUGA, D. (2009): "Psicología ambiental y calidad de vida". Observatorio ambiental. Vol. 12, 241-246.

Gupta, J. (2010): “A history of international climate change policy". Wiley Interdisciplinary Reviews Climate Change. Vol 1 (5), 636-653.

IPCC (1990): Climate Change: The IPCC Scientific Assessment. Cambridge: Cambridge University Press.

IPCC (1995): Working Group I. Summary for Policymakers: The Science of Climate Change.

IPCC (1995b): Climate Change 1995: Economic and Social, Dimensions of Climate Change. Cambridge: Cambridge University Press.

IPCC (2001): Climate Change 2001: The Scientific Basis, 2001. Cambridge: Cambridge University Press.

IPCC (2007): Climate Change 2007: The Physical Science Basis. Cambridge: Cambridge University Press.

MCMICHAEL, J.; FRIEL, S.; NYONG, A. Y CORVALÁN, C. (2008): "Global environmental change and health: impacts, inequalities, and the health sector". BMJ. Vol. 336, 191 - 194.

WMO (1979): Declaration of the World Climate Conference, Proceedings of the World Climate Conference: A Conference of Experts on Climate and Mankind, Geneva No. 537. ISBN: 92-63-10537-5.

WHO (2004): The world health report 2004: changing history. Geneva.

WHO (2008): Climate change and health: Resolution of the 61st World Health Assembly. SUNYER, J. (2012): "Promoción de la salud frente al cambio climático". Gac. Sanit. Vol. 24 (2), $101-102$. 